\title{
Jóvenes en la encrucijada de los nuevos tiempos. Una mirada a sus prácticas y consumos culturales desde la Universidad de Granma*
}

\author{
Yulianne Pérez Escalona', Wilfredo Manuel Castro Villa² \\ Universidad de Granma (Cuba)
}

Recibido: noviembre 12 de 2014 - Revisado: febrero 11 de 2015 - Aceptado: abril 29 de 2015

Referencia formato APA: Pérez, Y., \& Castro, W. M. (2015). Jóvenes en la encrucijada de los nuevos tiempos. Una mirada a sus prácticas y consumos culturales desde la Universidad de Granma. Revista Cientifica Guillermo de Ockham, 13(1), 127-134.

\section{Resumen}

La incesante multiplicación tecnológica y su penetración en todos los intersticios de la vida cotidiana debe ser punto de constante atención. Por ello, resulta necesario contribuir a la comprensión y explicación de cómo el mundo digital y las tecnologías inciden en los jóvenes, lo que da motivo a transformaciones socioculturales, fundamentalmente en sus prácticas y consumo. Se propone indagar en las prácticas y consumos culturales de los jóvenes de la Facultad de Ciencias Informáticas de la Universidad de Granma, de manera que esta investigación sirva de diagnóstico para conocer cómo se manifiesta este fenómeno en dicho contexto. El trabajo realizado responde a una de las acciones del proyecto de investigación La juventud cubana entre los modelos de inclusión y exclusión sociocultural: los consumos y prácticas culturales en jóvenes del oriente cubano (2012-2015).

Palabras clave: Jóvenes, prácticas culturales y consumo cultural

\section{Youths in the crossroads of modern times. A close look at their cultural practices and consumption from the University of Granma}

\section{Abstract}

The incessant technological multiplication and its incursion across the interstices of daily life must be a constant point of attention. Which is why it is necessary to contribute to the understanding and explanation of how the digital world and technologies affect young people, which gives reason to sociocultural transformations, mainly in their practices and consumption. The purpose is to investigate in the cultural practice and consumption of young people from the Computer Science Faculty in the University of Granma so that this research will serve as a diagnostic to know how this phenomenon manifests itself in this context. The work realized responds to one of the actions of the research project

* Este artículo se deriva del proyecto de investigación La juventud cubana entre los modelos de inclusión y exclusión sociocultural: los consumos y prácticas culturales en jóvenes del oriente cubano. (2012-2015). Centro de Estudios para el Desarrollo Integral de la Cultura (Cedic). Universidad de OrienteVenezuela.

1. Licenciada en Estudios Socioculturales de la Universidad de Granma. Profesora e investigadora de la Facultad de Ciencias Informáticas de Universidad de Granma. E-Mail: yperez@grm.uci.cu

2. Licenciado en Estudios Socioculturales de la Universidad de Granma. Profesor e investigador de la Facultad de Ciencias Informáticas de Universidad de Granma. E-Mail: wcastro@grm.uci.cu 
"Cuban youth between the models of sociocultural inclusion and exclusion: cultural consumption and practices in youths from eastern Cuba. (2012-2015).

Keywords: Youths, cultural practices and cultural consumption

\section{Juventude na encruzilhada dos tempos. Um olhar sobre as suas práticas e consumo cultural da Universidade de Granma}

\section{Resumo}

A tecnologia de multiplicação incessante e sua penetração em todos os interstícios da vida diária deve ser um ponto de atenção constante. Por isso, é necessário contribuir para a compreensão e explicação de como o mundo e as tecnologias digitais que afectam os jovens, o que dá motivo para mudanças socioculturais, principalmente em suas práticas e consumo. Propóe-se a investigar as práticas e consumo cultural de jovens da Faculdade de Ciência da Computação Universidade de Granma, então estas pesquisas, de diagnóstico servem para saber como este fenómeno manifesta-se nesse contexto. $\mathrm{O}$ trabalho responde às ações do projeto de pesquisa entre os modelos de inclusão de jovens cubanos e exclusão sociocultural: consumo cultural e as práticas em Cuba jovem oriental. (2012-2015)

Palavras-chave: Juventude, práticas culturais e de consumo cultural

\section{Introducción}

Muchas son las investigaciones que dan cuerpo conceptual a las categorías consumo y prácticas culturales y no son pocas las ocasiones en las cuales se profundiza en la interrelación que se produce entre ellas. Este abordaje teórico apunta en las últimas décadas a intensificar las miradas a la incidencia y redimensionamiento que se producen a partir de las nuevas tecnologías de la información y las comunicaciones (NTIC).

En los jóvenes recae, fundamentalmente, la atención de importantes teóricos, que establecen como principal espacio de consumo desde las NTIC a la familia. Sin embargo, un espacio que en la actualidad es cada vez más invadido por estas tecnologías para el desarrollo de sus procesos, es la universidad. Los niveles que han alcanzado los consumos y las prácticas culturales de los universitarios indican la necesidad de dar un nuevo giro a la dinámica del desarrollo de esta institución y de su colectivo.

La formación del personal en el campo de la informática en Cuba, tomó impulso a partir de la década de los setenta con la puesta en práctica de un plan en el marco del sistema nacional de computación, cuyo objetivo era preparar en el menor plazo posible analistas de sistemas, ingenieros de sistemas, operadores, técnicos de mante- nimiento y programadores. Su perfil los expone a un constante intercambio o interacción con las NTIC.

El estudio que se expone se lleva a cabo en la Facultad Regional de la Universidad de Granma, la cual fue creada con dos objetivos: informatizar la región y desarrollar la industria del software. El $100 \%$ de los estudiantes de cuarto y quinto ańo y cerca del $60 \%$ del resto, son incorporados a proyectos productivos e investigativos de software en interés y por encargo de la sociedad cubana, en campos como la educación, la salud, el deporte, el gobierno en línea, el software libre, los sitios y portales web, los productos multimedia la realidad virtual, entre otros. Ello pone en evidencia grandes niveles en cuanto a posibilidades de utilización de las NTIC y por ende, una mayor familiaridad de su población con estas.

En este sentido, se visualizan en la facultad actitudes y comportamientos que se han tornado comunes, como el auge masivo de la mensajería instantánea (chat, correos) y el desarrollo de blogs y redes. Con estas nuevas formas de relacionarse, los estudiantes se ausentan de la vida social, cambian sus horarios habituales y no tienen tiempo para conversar y relacionarse, a menos que no sea por la red. Se crea un mundo virtual en el cual la realidad social pierde espacios en las prioridades de los estudiantes y profesores.

$<$ Universidad de San Buenaventura, Cali - Colombia 
Se dan identidades temporales hacia diferentes enfoques (ser un personaje de película, serie o juego), que desaparecen una vez se gradúan y regresan a sus lugares de origen. Además, en el espacio virtual no se hace necesario identificarse físicamente, se pueden esconder las identidades individuales y mostrar solo aquellos elementos que constituyen los "nosotros" en ese espacio donde las identidades no tienen un carácter de permanencia que en el mundo real. Las nuevas formas de hacer las prácticas culturales mediante el mundo virtual que se crea en torno a la informática, por ejemplo la lectura, si no es digital no se desarrolla al igual que la escritura. Se crean comunidades virtuales especializadas de forma espontánea, es decir, formas de sociabilidad construidas en torno a intereses específicos. Por lo antes expuesto, se busca identificar los consumos y prácticas culturales de los jóvenes de la Facultad Regional de la Universidad de Granma.

Los datos arrojados invitan a profundizar en estos resultados ya no a partir de la observación pura del número, sino con el acompańamiento inteligente de su significación. Tendencias de consumos y prácticas culturales alcanzan el protagonismo como vía para un posterior análisis y con vista a ser más pertinente la utilidad de la información que se muestra. Para el desarrollo de la investigación, se tomaron como antecedentes los estudios de consumos en Cuba desde el año 1995 llevados a cabo por el Instituto de Investigación y Desarrollo de la Cultura Juan Marinello, las investigaciones de consumo en Latinoamérica que posibilitan contar con valiosa información teórica y metodológica y las encuestas nacionales aplicadas en varios países latinoamericanos como México (2004), Chile (2005) y Colombia, así como el material fáctico disponible.

\section{Metodología}

En la apropiación y uso de bienes, el consumo refleja significados y sentidos atribuidos a dichos bienes, en los que prevalece el valor simbólico sobre los valores de uso y de cambio. De esta manera, aflora como una construcción subjetiva en la que cada grupo social tiene demandas, motivos y actitudes propias hacia diferentes opciones culturales y perfila así identidades bien delineadas que responden a representaciones y realidades culturales propias. Hablamos aquí de una tendencia que indaga en el mundo de las significaciones y de la subjetividad, en la búsqueda de alternativas interpretativas y de un conocimiento profundo de causales y orientaciones que abarca la realidad tal y como es percibida y apropiada por los individuos, por lo cual resulta evidente la conveniencia de la investigación cualitativa.
El cuestionario fue aplicado a treinta estudiantes, de los cuales el $50 \%$ corresponde al sexo femenino; el rango de edad está entre los dieciocho y los veintiún años de edad. En cuanto al lugar de residencia, tres pertenecen a la provincia de Holguín, tres al municipio Palma Soriano, uno a las Tunas, uno a la provincia Granma y uno a la provincia Guantánamo. Diez estudiantes de la carrera de Filosofía con un rango de edad entre los dieciocho y los veinticuatro ańos, de los cuales cuatro pertenecen al sexo femenino y seis al masculino.

Para un análisis más detallado se hizo una diferenciación territorial donde se tomaron tres estudiantes de Guantánamo, tres de Granma, cuatro de Santiago de Cuba y diez estudiantes del cuarto ańo de la carrera de Historia del Arte. Sus edades edad oscilan entre los veinte y los veintidós años, de los cuales nueve pertenecen al sexo femenino y uno al sexo masculino. Seis son becados y cuatro externos, para un total de treinta encuestados. Las variables definidas para el estudio se corresponden con visitas a bibliotecas, museos, discotecas y teatros; actividades en el tiempo libre, género musical, práctica de deportes, comidas favoritas, programas audiovisuales y preferencias al vestir.

Los comentarios posteriores a la aplicación del instrumento estuvieron dirigidos a socializar las respuestas y cómo refirieron elementos en los que no habían pensado anteriormente.

\section{Concepto}

Múltiples han sido las dimensiones desde las cuales se ha abordado la categoría consumo. Los estudios de consumo cultural alcanzan protagonismo en el abordaje teórico hacia el entendimiento del comportamiento, los gustos, los intereses y los hábitos de determinados grupos humanos. Aun cuando se da tratamiento a esta categoría como tema de investigación en la agenda de los estudios culturales desde 1970, todavía a inicios de la década de los noventa estos eran insuficientes.

El consumo en sí y consumo cultural son conceptos que la sociología ha incorporado a sus desarrollos teóricos y en torno a ellos se han construido teorías que explican los complejos procesos de la realidad contemporánea. Esta idea permite aseverar que el fenómeno del consumo ha devenido en un tema de interés para todos aquellos que han reflexionado sobre las transformaciones de la modernidad, que si bien para algunos esta materia no ha llegado a constituirse en un marco conceptual sólido (Callejos, 1995; De Pablos, 2003; Sunkel 2002), los caminos 
recorridos desde la sociología clásica ofrecen sustantivos recursos para ubicar encuadres teóricos y metodológicos cuando de consumo y consumos culturales se trata: el fetichismo como mercancía (Marx; 2008); la ciudad como consumo (Weber; 1984); la moda en la cultura del consumo (Simel, 1988), la acumulación dineraria y clase ociosa, (Veblen, 2008), formas de intercambio económico como instituciones sociales (Mauss, 2009), el consumo en la interacción en los espacios del anonimato (Augé, 2000), el consumo como un modo de actividad sistemática y de respuesta global en el cual se funda todo nuestro sistema cultural (Baudrillard, 2009). Los consumos de los bienes significan antes que los propios bienes, los "burgueses bohemios (Featherstone, 1999). Sentidos por los usos que le dan forma social; Sunkel (2002) y Castells (1994).

Los desarrollos que ha alcanzado la sociología contemporánea en los últimos setenta años han abierto un campo disciplinar que centra sus análisis en la cultura, espacio donde el consumo aparece como concepto dentro del proceso de la reproducción social (Bourdieu, 2003). Con ello asistimos a un cambio de paradigma: el paso del consumo como alineación, al consumo como apropiación, al considerar al consumidor como un agente activo y los conceptos de usos, apropiaciones y estrategias adquieren connotaciones argumentativas.

Para los autores de este trabajo, el eje que ha permitido articular el desarrollo de los estudios sociológicos del consumo es la tríada usos-formas-estrategias y por consiguiente, el principal fundamento para exponer y argumentar el marco de explicación en el proceso de reproducción social. Con este encuadre, se explicitan el consumo y el consumo cultural y a su vez estos conceptos posibilitan encontrar respuestas a las nuevas prácticas de consumo culturales que comienzan a caracterizar una buena parte de nuestras sociedades.

Para Bourdieu, (2003)

Hablar de consumo cultural es decir que hay una economía de los bienes culturales, pero que esta economía tiene una lógica específica $[\ldots]$ es un momento de un proceso de comunicación, es decir, un acto de desciframiento, de decodificación que supone el dominio práctico o explícito de una cifra o de un código (p. 230).

Los estudios del consumo cultural han dado cuerpo a las nuevas realidades socioculturales, las cuales se construyen en los espacios sociales como elementos activos en la experiencia cotidiana de la gente y como escenarios que aparecen entre la ciudadanía y las instituciones. Se condensan las posibilidades y las problemáticas de la ciudad como referentes de identidad urbana. Sin embargo, debemos ampliar el conocimiento y profundizar la investigación sobre los usos y apropiaciones de los consumos dentro de la trama de relaciones, prácticas, actores y formas organizativas que surgen en el espacio social.

En el consumo cultural están involucrados no solo la apropiación en sí, sino también las variables de los usos sociales, la percepción-recepción, el reconocimiento cultural y la "construcción" de ciudadanía en sentido de pluralidad y por tanto, de concepción democrática de la vida.

Dentro de los estudios mencionados ocupan un espacio fundacional los de García-Canclini (1999) y Martín-Barbero (2009). Si bien cada uno hizo aportes claves para llegar a una aproximación del concepto que se hace mención, su principal mérito radica en despertar con su reflexión el interés por las investigaciones sobre esta temática.

En su artículo, García Canclini (1999) luego de hacer un análisis de seis modelos a través de los cuales se trabaja el consumo y llegar a la conclusión de que ninguno es autosuficiente para abordar este problema, plantea que el consumo cultural es el "conjunto de procesos de apropiación y usos de productos en los que el valor simbólico prevalece sobre los valores de uso y de cambio o donde al menos estos últimos se configuran subordinados a la dimensión simbólica” (p. 34).

Por otro lado, Martín-Barbero (1987) plantea que

[...] el consumo no es solo reproducción de fuerzas, sino también de producción de sentidos: lugar de una lucha que no se agota en la posesión de los objetos, pues pasa aún más decisivamente por los usos que les dan forma social y en los que se inscriben demandas y dispositivos de acción que provienen de diferentes competencias culturales (p. 231).

Ambos autores en sus definiciones manifiestan elementos que si bien se enuncian de diferentes maneras, son comunes. Dentro de estos se encuentra que el aspecto material más que determinante en el consumo pasa a un segundo plano subordinado al significado que se le atribuye socialmente.

Como ya se mencionaba, prácticas culturales es un concepto ligado muy estrechamente al abordado con anterioridad, pues sus puntos de confluencias han permitido un desarrollo casi proporcional de ellos. Se pudieran escribir varios renglones sobre el tratamiento conceptual que se le ha dado a la categoría, pero solo se van a mencionar algunos autores que sin duda resultan eslabones de obligada referencia. Dentro de ellos se encuentran Martín-Barbero, Bourdieu y Giddens. 
Se asumen, entonces, las ideas del grupo de investigadores del Cedic que plantean que las prácticas culturales se manifiestan en el accionar del individuo, las colectividades, las instituciones en la vida cotidiana y el molde cultural, y adquieren significados en los marcos de la sociedad. Este mismo grupo reafirma el concepto con base en la sociología de la cultura y la antropología, referente a la forma general y a la manera de hacer y de actuar.

Tanto el consumo como las prácticas culturales se han redimensionado gracias al auge alcanzado por las NTIC. Los enfoques que se han generado para conceptualizarlas dirigen su mirada a entenderlas como "el conjunto de procesos y productos derivados de las nuevas herramientas (hardware y software), soportes de la información y canales de comunicación relacionados con el almacenamiento, procesamiento y transmisión digitalizados de la información" (González-Soto et al., 1996, citado en Ferro-Soto, Martínez \& Otero 2009, p. 2).

Con un análisis menos técnico y más enfocado al resultado o producto de las NTIC, Barbero (2009) afirma que la tecnología remite hoy en día a nuevas formas de percepción, lenguaje, sensibilidades y escrituras. La tecnología deslocaliza los saberes y modifica tanto el estatuto cognitivo como institucional de las condiciones del saber y las figuras de la razón, lo que conduce a un emborronamiento de las fronteras entre razón e imaginación, saber e información, naturaleza y artificio, arte y ciencia, saber experto y experiencia profana.

Diferentes estudios vinculan a los jóvenes como centro del impacto de las NTIC (Mato, 1994; Wortman, 2000; García-Canclini, 1999; Martín-Barbero, 2003; 2009; Margulis y Urresti, 1998). Los jóvenes, dadas sus características en los órdenes psicológico, sociológico, biológico, etc., están predispuestos a asumir las NTIC y originar cambios radicales en cuando a sus modos y estilos de vida. Establecen, entonces, una escala de significados con múltiples determinaciones que a pesar de su carácter activo al consumir o rechazar el producto cultural y la forma y el medio para hacerlo, los condicionan.

Los estudios sobre juventudes han definido este sector de la población de múltiples maneras. Toman como punto de partida diferentes criterios, dentro de los que se encuentran la edad, las diferencias generacionales a partir del consumo masivo de productos e imágenes de belleza que refieren la juventud a la condición del cuerpo y los criterios morales (Margulis \& Urresti, 1998).

Como aproximación al concepto de juventud, se trabajan dos conceptos empleados en el estudio de Domínguez
(2005). Una etapa de afianzamiento de las principales adquisiciones logradas en períodos anteriores. En la Juventud comienza a formarse una concepción teórico-filosófica de la realidad, sobre la base de todas las adquisiciones del desarrollo precedente, expresándose en la búsqueda del sentido de la propia existencia y en la elección del futuro lugar a ocupar en el entramado social. Se consolidan las formaciones motivacionales complejas, tales como la autovaloración y los ideales. Todo este sistema de necesidades, motivos y aspiraciones, se integra a la concepción del mundo, formación típica del período juvenil, la cual representa el nivel superior de integración de lo cognitivo y lo afectivo en la personalidad. En la juventud culmina, en lo esencial, el proceso de formación de la personalidad.

Una categoría histórico concreta que designa un grupo sociodemográfico internamente diferenciado según su pertenencia a la estructura social de la sociedad, constituyendo así su elemento más dinámico y móvil. No está biológicamente determinada -aunque se enmarca en determinados rangos de edades- sino definida socialmente por la naturaleza de la actividad que se desarrolla en esa etapa, lo que condiciona un conjunto de relaciones sociales específicas que conforman la condición juvenil a partir del significado propio de la etapa, la cual genera una identidad juvenil que es tanto autoidentidad, como identidad reconocida por el resto de las generaciones. En el marco de esas relaciones sociales, se alcanza la madurez física y sexual, se estructura y consolida la concepción del mundo como formación motivacional compleja, se desarrolla el carácter activo de la personalidad a través de la autodeterminación y se alcanza la madurez de las funciones laborales, familiares, sociales y políticas, para todo lo cual resulta determinante el contenido de la experiencia vital acumulada. Por ello, los límites de la edad juvenil, así como las divisiones internas del grupo, tienen un carácter histórico-concreto (Domínguez, 1994).

\section{Análisis de los resultados}

En lo que respecta al caso de los de Guantánamo, a dos de los tres encuestados les gusta pasear y al otro escuchar música. A cada uno le gustan géneros musicales diferentes como el rap, el hip-hop y el reguetón y van a la biblioteca una vez al mes. Dos prefieren leer libros y el otro periódicos. A los tres les gusta sacar fotografías, dos de ellos plantean que les gusta vestir a la moda y el otro que le gusta tener su estilo personal; uno expresa que le gusta vestir igual en la escuela y en la casa porque eso lo ayuda a sentirse más seguro de sí mismo. Los otros expresaron que no, porque la escuela no es lugar para exhibir 
la moda. A dos le gusta ir al cine y al otro al teatro. A dos de ellos les gusta practicar deporte y al otro no, pero los tres concuerdan con que les gusta mirar los deportes. Dos manifestaron su gusto por las películas y el otro por las series. A dos les gusta comer espaguetis y al otro dulces. Dos de ellos expresaron su gusto por los vinos y el otro por el ron. A ninguno le gusta fumar y consideran importante esta investigación, porque ayuda a definir los gustos de la juventud universitaria en la actualidad. Como vimos anteriormente, se hizo un análisis a partir de los gustos y preferencia de los jóvenes pertenecientes a la provincia de Guantánamo y se encontró que sus gustos se relacionan en un $80 \%$.

El análisis a partir de los tres estudiantes pertenecientes a la provincia de Granma, mostró lo siguiente: sus gustos son parecidos: escuchar música y comer. También se relacionan con el tipo de música que han de escuchar, pues tienen una inclinación por el género romántico. Visitan la biblioteca una vez a la semana y les gusta leer revistas. De ellos uno toca un instrumento y los otros dos hacen trabajos con fines creativos y un estilo propio. En lo referente a la manera de vestir, expresan no vestir de la misma manera en la escuela que en la casa. Uno de ellos explica que en su casa se pone ropas extremadamente cortas que no usa en la escuela. A dos de ellos les gusta ir a exposiciones y al otro al teatro. Los tres practican deportes pues es muy bueno para la salud. Sus deportes favoritos son el béisbol, el fútbol y el ciclismo y sus programas preferidos son las películas. En cuanto a las comidas y bebidas, les gusta el marisco, los chatinos y los dulces. Respecto de las bebidas, sus favoritas son las cremas y los vinos. No fuman, pues consideran que provoca mal aliento. Por último, plantean que esta investigación es importante.

Los últimos cuatro encuestados de la provincia de Santiago de Cuba manifiestan su gusto por ir a la playa en su tiempo libre. Uno de ellos se inclina por el rock, otro por la trova y los dos últimos por el reguetón. En la encuesta aplicada a estos jóvenes, se pudo evidenciar que no asisten a la biblioteca, pues prefieren leer otro tipo de libros. Les gusta sacar fotografías. A dos les gusta tener su estilo propio y a los otros vestir a la moda (no visten de la misma manera en la escuela y en la casa, porque son escenarios diferentes). A uno le gusta ir a peñas de rock y a otro disfrutar de artes visuales. Los demás prefieren ir a discotecas. A uno de ellos no le gusta practicar deportes, pero al al resto sí (béisbol, baloncesto y voleibol). A uno le gusta los muńequitos, al otro las series y al otro los deportes. En cuanto a las comidas, expresan no tener preferencia por alguna en especial. Fuman, pero no por vicio y consideran importante la investigación porque les permite tener una visión real de los gustos y preferencias de las personas, principalmente en una edad como la de ellos cuando no se tiene estabilidad en nada y mucho menos a la hora de elegir algo determinado.

En la guía de observación se pudo comprobar que los estudiantes de filosofía no tienen un estilo de vida diferente al de los demás y consumen lo mismo. Su manera de vestir y expresarse es muy parecida a la de los restantes estudiantes universitarios. También se pudo constatar que necesitan más integralidad y responsabilidad con su carrera y darle distinción, pues será su futura profesión.

Un análisis porcentual de la muestra señala que con respecto a las actividades que efectúan en el tiempo libre, al $40 \%$ le gusta leer, escuchar música y visitar a amigos; al $90 \%$ ver la televisión; al $50 \%$ dormir y pasear y por último, al $30 \%$ bailar, comer e ir a la playa. En cuanto a las actividades que realizan en su tiempo de ocio, el $20 \%$ prefiere leer, el $30 \%$ ver la televisión y viajar para conocer lugares nuevos, el $70 \%$ ir a la playa y el $60 \%$ visitar a sus familiares.

En cuanto al sexo femenino, el $80 \%$ manifestó preferencia por el género musical romántico; el $20 \%$ por el rock; el $40 \%$ por la música popular bailable y el $30 \%$ por el reguetón y la trova. En referencia a la frecuencia con que asisten a la biblioteca, el $30 \%$ asiste una vez al ańo, el $20 \%$ una vez al mes y el restante $50 \%$ una vez a la semana. En lo tocante a la forma de vestir, el $80 \%$ manifestó tener un estilo propio y el 20 \% restante expresó vestir a la moda o combinar ambas tendencias. Por otra parte, el $70 \%$ manifestó que viste de igual forma en la escuela y en la casa, dado que gusta de conservar un estilo propio en todos los lugares y sentirse ellas mismas. Otras plantearon no tener preferencias, pues se sienten de igual manera en ambos lugares. El restante $30 \%$ planteó vestir de forma diferente, porque el contexto no es el mismo. La ropa de la escuela es de diario, cómoda y correcta y la de la casa es informal, además de corta (la de la escuela es más larga). Con respecto a sus gustos, al $40 \%$ le gusta ir al cine, al $60 \%$ a espectáculos de danza, al $30 \%$ a las discotecas y a los museos.

De las diez encuestadas, el $60 \%$ planteó que le gusta practicar deportes y el restante $40 \%$ no. Dentro de los tipos de deportes que prefieren, están el béisbol, el voleibol, el ajedrez y el futbol. Dentro de los tipos de programas audiovisuales, el $20 \%$ prefiere la novela. El $100 \%$ manifestó ver películas, el $80 \%$ series, el $40 \%$ muñequitos y el $20 \%$ documentales. En cuanto a sus comidas favoritas, el $80 \%$ prefiere los mariscos, el $60 \%$ las ensaladas, el $40 \%$ los potajes, moros y cristianos, el 
$20 \%$ el arroz y los chatitos, el $70 \%$ las pizzas y dulces y el $50 \%$ los espaguetis, los lácteos y las carnes.

El $70 \%$ prefiere como bebidas la cerveza, el $50 \%$ los vinos, el $20 \%$ las cremas y licores, el $90 \%$ los jugos y el $80 \%$ los batidos. El $30 \%$ planteó su gusto por fumar y el restante $70 \%$ no, porque daña la salud y el ambiente. Lo consideran desagradable y afecta de cierta manera el aspecto.

Por último, el $100 \%$ de los encuestados considera importante la investigación sobre las prácticas y el consumo cultural en los jóvenes, pues permite la promoción y prevención con respecto al consumo excesivo y constituye un indicador para medir por dónde anda la sociedad cubana, sus retos y sus perspectivas.

Varias son las lecturas que se obtienen de estos resultados. En primer lugar, se evidencia la intensa dinámica cultural que hoy viven los jóvenes universitarios, en la que practicar deportes, asistir a las discotecas, ver películas e intercambiar entre amigos, entre otros gustos, constituye el centro de los consumos culturales en este escenario. Por otro lado, se evidencia cómo a pesar de ser estudiantes universitarios, no tienen concebido dentro de sus principales prácticas la lectura y la visita a bibliotecas, pues el $30 \%$ asiste una vez al ańo, el $20 \%$ una vez al mes y el restante $50 \%$ una vez a la semana y ubican en un mayor nivel de prioridad las actividades mencionadas anteriormente. Por otro lado, en cuanto a la diferenciación territorial no existen diferencias significativas en torno a los consumos en los jóvenes universitarios, lo cual evidencia un comportamiento homogéneo respecto de la jerarquización de sus gustos. En este sentido, cabe concluir que las formas de consumir de estos jóvenes no están en función del tipo de carrera que estén estudiando ni de la diferenciación regional, sino del lugar donde pasan la mayor parte del tiempo y donde transcurre su vida social -la residencia estudiantil- y por los efectos directos e indirectos de una globalización cultural.

Los resultados anteriores denotan el evidente interés de nuestros jóvenes universitarios por una variedad de prácticas. Aunque aspiremos a que los educandos de esta especialidad promuevan el culto al arte y se formen con habilidades de interpretación de los procesos culturales, llama la atención la amalgama de sus prácticas, las cuales denotan que el contexto sociocultural puede influir, pero no determina la preferencia por una práctica $u$ otra. Los significados que otorgan los estudiantes a su consumo cultural, están relacionados con buscar nuevas amistades, presumir de las prendas (ropas, zapatos de marca o de precios elevados, cadenas, relojes, dinero etc.), reafirmar identidades (Miki y los Friki), trascender los planes existenciales de su cotidianidad y lograr el reconocimiento social.

En cualquier análisis que se pretenda hacer de los jóvenes universitarios, no puede olvidarse que este grupo forma parte también de un grupo mayor, a saber, la juventud toda y de manera general la sociedad. El consumo cultural que muestran, responde a una cuestión generacional que tiene que ver con las tendencias de la moda o el esteticismo que prevalece en los jóvenes. Es decir, el consumo que exige lo bonito, lo divertido, la imagen, lo brilloso etc., y todas aquellas publicaciones -en el caso de la lectura, por ejemplo-, que se alejen de estos elementos son poco atractivas para los intereses de los jóvenes lectores. Prevalece el consumo en el que el joven no tiene que hacer esfuerzo alguno intelectual, porque todo está diseñado de forma simple y coloquial que no desarrolla el capital lingüístico y estético de la persona. Lo peor no es que consuman este tipo de productos, sino que sea lo único que consuman.

Lo que motiva el consumo cultural de los jóvenes, está asociado a aspectos subjetivos, como el crecimiento espiritual y profesional y hacer nuevas relaciones. Ello se corresponde con el grupo en el que están insertos, donde socializar y compartir con los otros es más demandado que en cualquier otra etapa de la vida social.

Los elementos que nos proporcionaron la encuesta y las observaciones realizadas, no difieren de otros que pueden hallarse en estudios análogos. En todo caso, permiten reafirmar por dónde transitan los consumos culturales de los jóvenes universitarios en estos años del siglo XXI. De allí que un asunto de interés para el análisis es reflexionar acerca de las relaciones de los jóvenes universitarios con esos consumos: la influencia del contexto sociohistórico en la elaboración de los significados de los consumos y la vinculación de dichos consumos con la construcción de la vida cotidiana.

\section{Conclusiones}

La investigación confirma que el consumo de los jóvenes universitarios está determinado por diversos factores, entre los que se destacan los aspectos culturales, lo cual implica que la cultura le da un especial significado a los productos cotidianos. Las formas de consumir de estos jóvenes no están en dependencia del tipo de carrera que estén estudiando ni de la diferenciación regional, sino del lugar donde pasan la mayor parte del tiempo y en el que transcurre su vida social -la residencia estudiantil- y 
por los efectos directos e indirectos de una globalización cultural.

Los resultados arrojados en el diagnóstico y su correspondiente análisis, indican que hay que originar cambios en la manera de desarrollar los procesos universitarios, de forma que esa comunicación lineal entre el profesor y el estudiante asuma nuevos matices que sustituyan la unidireccionalidad del proceso y tengan en cuenta las diferentes fuentes de información por las cuales se nutren los educandos.

De igual manera, los resultados permiten evaluar las políticas culturales de las instituciones culturales de la provincia de Granma.

\section{Referencias}

Augé, M. (2000). Los "no lugares” espacios del anonimato: una antropología de la sobremodernidad. Barcelona: Gedisa.

Balardini, S. (2006). Jóvenes, tecnología, participación y consumo. Recuperado de http://goo.gl/LoVSRc

Baudrillard, J. (2009). La sociedad de consumo sus mitos, sus estructuras. Madrid: Siglo XIX.

Bermúdez. E. (Septiembre, 2001). Consumo cultural y representación de identidades juveniles. Ponencia presentada en el congreso Latin American Studies Association, Washington.

Bourdieu, P. (2003). Creencia artística y bienes simbólicos: elementos para una sociología de la cultura. Buenos Aires. Grupo editorial Aurelia-Rivera.

Callejo, M. J. (1995). La construcción del consumidor global. Revista de ciencias sociales, 126, 77-96.

Castells, M. (1994). Flujos, redes e identidades: Una Teoría crítica de la sociedad informacional. En M. Castells, et ál. (Eds.), Nuevas Perspectivas criticas en Educación. Barcelona: Paidós.

De Pablo, J. (2003). La educación como motivo de reflexión. En J. De Pablo (Coord.), La tarea de educar: de qué hablamos cuando hablamos sobre educación (pp. 15-48). España: Biblioteca nueva.

Domínguez, L. (2005). Psicología del desarrollo. Problemas, principios y categorias: Universidad de La Habana.

Domínguez, M. I. (1994). Socialización y subjetividad juvenil. Revista cubana de psicología, 2(3), 109-116.

Dussel, I., \& Quevedo, L. (2010). Educación y nuevas tecnologías: los desafíos pedagógicos ante el mundo digital. Buenos Aires: Editorial Santillana.

Ferro, C., Martínez, A. I., \& Otero, C. (2009). Ventajas del uso de las tics en el proceso de enseńanza y aprendizaje desde la óptica de los docentes universitarios españoles. Revista electrónica de tecnología educativa, 29, 1-12.

García-Canclini, N. (1999). El consumo cultural: una propuesta teórica. En G. Sunkel (cord.), El consumo cultural en América Latina. Construcción teórica y lineas de investigación. Bogotá: Andrés Bello.

Giddens, A. (2010). Sociología. Madrid: Alianza editorial.

Gil, A., Feliu, J., Rivero, I., \& Gil, E. (2003). ¿Nuevas tecnologías de la información y la comunicación o nuevas tecnologías de relación? Niños, jóvenes y cultura digital. Barcelona: Universitat Oberta de Catalunya.

Margulis, M., \& Urresti, M. (1998). La construcción social de la condición de juventud. En L. Toscano et al. (Eds.). Viviendo a toda. Jóvenes, territorios culturales y nuevas sensibilidades (pp. 1-22). Santa Fe de Bogotá: Siglo del Hombre Editores.

Martín-Barbero, J. (1987). De los medios a las comunicaciones. Comunicación, Cultura y Hegemonía. Barcelona: Editorial Gustavo Gili.

Martín-Barbero, J. (2003). Saberes hoy: diseminaciones, competencias y transversalidades. Revista Iberoamericana de Educación, 32. Recuperado de http://goo.gl/q1VVif

Martín-Barbero, J. (2009). Cuando la tecnología deja de ser una ayuda didáctica para convertirse en mediación cultural. Revista Teoría de la Educación, 10(1). 19-31.

Marx, K. (2008). El capital. Crítica de la economía politica. México: Siglo XXI

Mato, D. (2002). Estudios y otras prácticas intelectuales latinoamericanas en cultura y poder. Caracas: CLACSO.

Mauss, M. (2009). Ensayo sobre el don. Forma y función del intercambio en las sociedades arcaicas. Buenos Aires: Katz Editores.

Morduchowicz, R. (2008). Significados, consumos y prácticas culturales de los jóvenes. Buenos Aires: Paidós.

Simmel, G. (1988). La aventura. Barcelona: Península.

Sunkel, G. (2002). Una mirada otra. La cultura desde el consumo. En D. Mato (Coord.), Estudios y otras prácticas intelectuales latinoamericanas en cultura y poder (pp. 287294). Caracas: CLACSO.

Terrero, P. (2006). Ocio, prácticas y consumos culturales. Aproximación a su estudio en la sociedad mediatizada. Recuperado de http://goo.gl/WBukei

Veblen,T. (2008). Teoría de la clase ociosa. Espańa: Alianza Editorial.

Weber, M., (1984). Economía y Sociedad. Esbozo de sociología comprensiva. México DF: Fondo de Cultura Económica.

Wortman, A. (Junio, 2000). Identidades sociales y consumos culturales: el consumo de cine en la Argentina. Trabajo presentado en Crossroads in Cultural Studies 3rd International Conference, Birmingham, UK.

$134<$ Universidad de San Buenaventura, Cali - Colombia 